\title{
Optimization in Cooperative Spectrum Sensing
}

\author{
Ju Chan $\mathrm{Na} 1)$
}

\begin{abstract}
An encouraging technology that grabs the clash between spectrum scarcity and underutilization is cognitive radio (CR), spectrum sensing is the main task of cognitive radio. Cooperative spectrum sensing overcome the adverse effect of shadowing and multipath. In this paper we are focusing on the optimization of cooperative spectrum sensing. Optimization strategy is proposed in order to optimize the overall performance by variation of SNR. In this technique we consider three parameters, spectrum sensing technique used locally, SNR and the number of users. Decision made by all the users is sent to the fusion center which estimate the final presence or absence of primary user.
\end{abstract}

Keywords: cognitive radio, $\mathrm{CR}$, spectrum sensing, secondary users, primary users, cooperative spectrum, optimization, fusion centre.

\section{Introduction}

Radio spectrum is the backbone of the wireless communication due to its role of providing the transmission medium. But in this era a lot of spectrum has been occupied as a result there is scarcity of radio spectrum. Cognitive radio has provided the solution of this problem. All the licenses users do not occupy the spectrum all the time. According to the U.S. Federal Communications Commission, the allocated spectrum resources are severely underutilized in massive temporal, spatial, and spectral dimensions[1]. This is mainly because under existing governing policies, frequency bands are statically assigned to licensed/primary users (PUs), and no reutilization is permitted for unlicensed/secondary users (SUs). In general, spectrum sensing solutions can be categorized as cooperative and non-cooperative[2]. More specifically, cooperative solutions depend on multiple SUs to exchange spectrum occupancy information through individual local measurements. The existing studies specify that association among SUs improves the efficiency of spectrum utilization, and allows reduction of the constraints at

Received(October 26, 2016), Review Result(1st: November 11, 2016, 2nd: December 7, 2016), Accepted(March 10, 2017)

${ }^{1}$ (Corresponding Author) Department of Electronic Engineering, Gachon University

email: chan2233@naver.com 
individual SUs. Spectrum sensing is a key function of cognitive radio to inhibit the harmful interference with licensed users and identify the available spectrum for improving the spectrum's consumption. However, detection performance in practice is often compromised with multipath fading, shadowing and receiver uncertainty issues[3]. To moderate the impact of these issues, cooperative spectrum sensing has been shown to be an operative method to improve the detection performance by developing spatial diversity.

The essential task of each CR user in CR networks, in the most basic sense, is to detect the licensed users also known as primary users (PUs), if they are present and identify the available spectrum if they are absent. This is usually achieved by sensing the RF environment, a procedure called spectrum sensing[4]. The objectives of spectrum sensing are twofold: first, CR users should not cause destructive interference to PUs by either switching to an available band or restraining its interference with PUs at an acceptable level and, second, CR users should efficiently identify and exploit the spectrum holes for necessary throughput and quality of service (QoS).

Spectrum sensing is essential for CR networks as it detects the state of channel for opportunistic reutilization[5]. There are two important metrics in spectrum sensing:

(1) detection probability, and

(2) false alarm probability[6].

The higher the better the PUs are protected, the lower the more efficiently the channel can be reutilized by SUs. To increase the detection probability, a collection of signal detection techniques are proposed in [7-9][18-25].

\section{Sensing and Result}

\subsection{Spectrum Sensing}

In this scenario we have considered a cognitive radio network compose of $\mathrm{K}$ CRS (Secondary users) and a common receiver. In this network each cognitive radio sense the spectrum in dependently and decision made locally is sent to the common receiver which fuses all the decision statistics to infer the absence or presence of the PU. The binary hypothesis is represented as:

$\mathrm{H} 0$ : primary user is absent;

$\mathrm{H} 1$ :primary user is present 
This hypothesis consider the spectrum sensing in the CR. The sensing model is to decide between the following two hypothesis[6],

$$
X_{i}(t)= \begin{cases}W_{i(t)} & H_{0} \\ H_{i}(t) S(t)+W_{I(t),} & H_{1}\end{cases}
$$

Where received signal is represented by $\mathrm{Xi}(\mathrm{t})$ ati time slot.

$\mathrm{S}(\mathrm{t})$ is the PU signal, $\mathrm{Wi}(\mathrm{t})$ is represented as the additive white Gaussian noise (AWGN), and is the channel gain of the path between $\mathrm{CR}$ and the primary user. We assume that the sensing time is smaller than the coherence time of the channel. Then, the sensing channel can be viewed as time-invariant during the sensing process. Without loss of generality, we denote as Hi. For the ith cognitive radio the probability of detection and probability of false alarm in AWGN channel is represented as [4].

$$
\begin{gathered}
P_{f, i}=\frac{r\left(u, \frac{\lambda_{i}}{2}\right)}{r(u)}(1) \\
P_{d, i}=Q_{u}\left(\sqrt{2 r_{i}}, \sqrt{\lambda_{i}}\right)
\end{gathered}
$$

And $P_{m, i}=1-P_{d \cdot i}$

In the above equation, $\lambda_{i}$ and $\gamma_{i}$ denote the energy detection threshold and signal to noise ratio at the $\mathrm{CR}$ respectively, $\mathrm{u}$ is the time bandwidth product of the energy detector.

$$
\tau(a, x)=\int_{x}^{\infty} t^{a-1} e^{-t} d(t)
$$

\subsection{Type of Cooperative Sensing}

A. Centralized sensing: In this methodology to $C R$ cooperative spectrum sensing, there is a central CR called fusion centre (FC) within the network that collects the sensing information from all the sense CRs within the network. For data cooperative, all CRs are altered to a control channel where a physical point-to-point link between each cooperating CR and the FC for sending the sensing results is called a broadcasting channel as shown in Figure (1-a). FC then analyses the information and regulates the bands that can and cannot be used. 


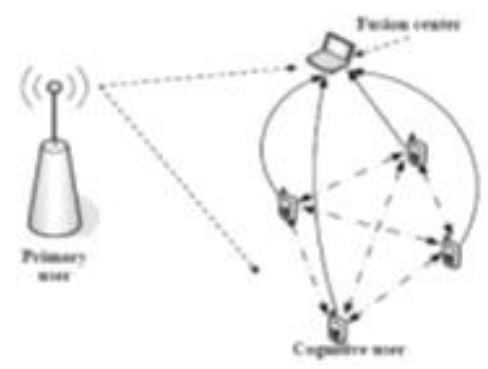

[Fig. 1-a] Broadcasting Channel

B. Distributed sensing: Unlike centralized approach, distributed cooperative sensing does not rest on a FC for making the cooperative decision. Using the distributed approach for $\mathrm{CR}$ cooperative spectrum sensing, no $\mathrm{CR}$ takes control. Each $\mathrm{CR}$ sends its specific information of sensing to other CRs and fuses its data with the received data of sensing, and decides whether or not the PU is present by using a local condition as shown in Figure(1-b). However this approach requires for the individual $\mathrm{CRs}$ to have a much upper level of independence, and possibly setting themselves up as an ad-hoc network.

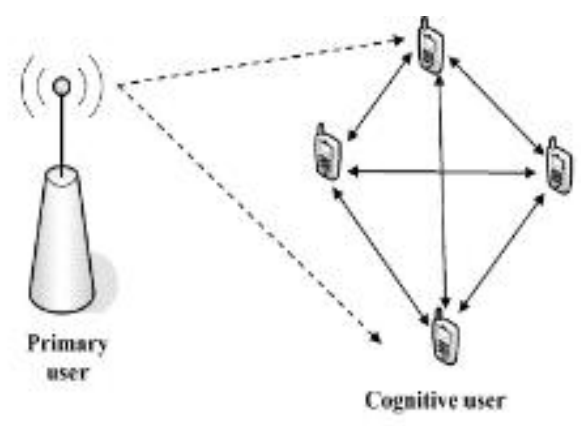

[Fig. 1-b]: Cognitive User

\subsection{Concept of optimization}

There are two type of decision rule that are used to detect the presence of the primary user, Soft decision rule and hard decision rule. In this paper we use the hard decision rule. Every user in the cooperative system detect the presence of the primary user and derive a decision in the form of bit either 1 or 0 . If user detect the presence of the primary user it detect 1 or the absence is presented in form of 0 . All the users send their decision to the fusion center known 
as base station. All these decision are combined together to derive a final result with AND or OR rule. Different schemes and factors have been investigated to optimize the hard cooperative sensing performance by minimizing the total error probability, or exploiting the probability of detection [4][7-8][16-18]. It was achieved by optimizing the number of cooperated Cognitive radios and the threshold. The author has choosen the total probability of detection in "OR" and "AND" fusion rules to peak by fixing the total false alarm probability In [7].

To decrease the total error probability under Neyman Pearson, and Bayesian criterions different strategies have been adopted in [19]. We assume that, compared with the distance from any cognitive radio to the primary transmitter, the distance between any two cognitive radios is trivial[4], so that the received signal at each cognitive radio experiences almost same path loss.

\subsection{Optimization of cooperative spectrum sensing}

In this section we are considering the optimization of cooperative communication when energy detection and decision fusion with hard cooperative spectrum sensing is applied. In the hard decision fusion scheme, local decisions of the nodes are sent to the decision maker. Cooperative sensing is done by fusion the sensing data of individual secondary users and make a final decision at the secondary BS. To minimize the transmission overhead of the sensing data, every secondary users will make their own sensing decision and transmit their one-bit decision to the secondary BS for fusion The main advantage of this method is the fact that it needs limited bandwidth [17]. We have a number of $G$ (or $r=1,2$. . K) CRs in the $\mathrm{CR}$ network, where each $\mathrm{CR}$ performs spectrum sensing locally using Energy Detection. Each CR transceiver is maintained by (N-IFFT/FFT) processers to perform both tasks of communication and sensing the environment. The primary transmitter with $\mathrm{N}$ subcarriers (N-IFFT/FFT) transmits OFDM-QPSK signal with energy over each sub carrier, and Ts which is symbol duration [1]. So, each CR estimates the power within each subcarrier in the frequency domain, with $=0,1 / \mathrm{N}, 2 / \mathrm{N} . . \mathrm{N}-1 / \mathrm{N}$ are the bins of normalized frequency. We assume that the total number of user $\mathrm{K}$ is fixed[1] .The optimal voting rule for cooperative spectrum sensing that minimize + is describe as follows. Suppose $G$ is a function given by

$$
G(n)=\sum_{l=n}^{k}\left\{\begin{array}{l}
k \\
l
\end{array}\right)\left[P_{f}^{L}\left(1-P_{f}\right)^{k-l}-\left(1-P_{f}\right)^{l} P_{m}^{k-1}\right]
$$


From equation (3) and (4) we get the total error probability

$$
Q_{\text {error }}=Q_{f}+Q_{m}=1+G(n)
$$

자 know that to get the maximum value of a curve can be obtained by differentiating it, so

$$
\begin{aligned}
& \frac{\partial G(n)}{\partial n} \approx G(n+1)-G(n)=\left[\left(1-P_{m}\right)^{n} P_{m}^{(k-n) P_{f}^{n}}\left(1-P_{f}\right)^{(k-n)]}\right. \\
& \left(\frac{k}{n}\right)\left[\left(1-P_{m}\right)^{n} P_{m}^{k-n}-P_{f}^{n}\left(1-P_{f}\right)^{k-n}\right]
\end{aligned}
$$

The optimal value of is obtained, when $\frac{\partial G(n)}{\partial} n=0$

$$
\left(1-P_{m)}^{n} P_{m}^{(k-n)}=P_{f}^{n}\left(1-P_{f}\right)^{(k-n)}\right.
$$

we get $n \approx\left[\frac{k}{(1+\alpha)}\right]$

$$
\text { where } \alpha=\frac{\frac{\ln P_{f}}{1-P_{m}}}{\frac{\ln P_{m}}{1-P_{f}}}
$$

\subsection{Result}

Local spectrum sensing : In this section we are examining the performance optimization of cooperative spectrum when local spectrum sensing is used .We have considered different frequencies will be computed at a specific frequency bin fi.. Fig. 2 shows the total error probability () versus the chosen local threshold for SNR $=10 \mathrm{db} \& \mathrm{~g}=5$ by monte-carlo simulation method using $\mathrm{ED}$ technique. Let us take $\mathrm{k}=10$ number of CRs cooperate the spectrum sensing decision at a CR-BS, in the CR's network. We have taken $\mathrm{SNR}=10 \mathrm{db}$ \& L=10 sample (i.e OFDM BLOCKS) are used locally for sensing[18] .In the fig shows the total error probability () versus the chosen local threshold for different number of $n$ out of $k$ CRs that controls the fusion rule in using ED technique. If we compare the different curves that signify the total error for different numbers of $\mathrm{n}$ in Fig. 3, we observe, there are visible difference in the performance through using $\mathrm{n}=1$ to 10 as a $\mathrm{k}=10$ fusion rule. Here, $\mathrm{n}=10$ which represent "AND" fusion rule, gives great total error associated to the other curves; it is 
found that $n=5$ gives the minimum total error (min Qerror) at the Equal values of SNR and threshold. Hence, $\mathrm{n}=5$ is the optimal fusion rule here (i.e., $\mathrm{n}$ optimal=2). The increase in SNR causes decrease in the min with variation in number of CRs.

Furthermore, for fixed SNR if the number of the total co-operated CRs, k, is increased above optimal then the is increased.

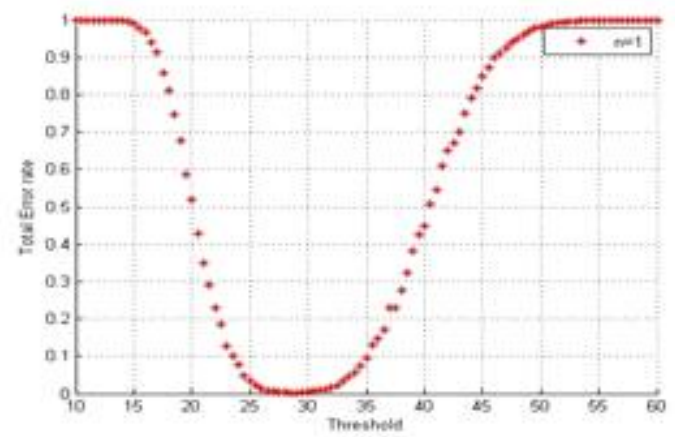

[Fig. 2] Total error probability ( $Q_{\text {error }}$ ) for $n=5$ CRs versus local threshold when ED is used locally with $\mathrm{SNR}=10 \mathrm{db}$ and $\mathrm{L}=10$ sensed samples used at each CR.

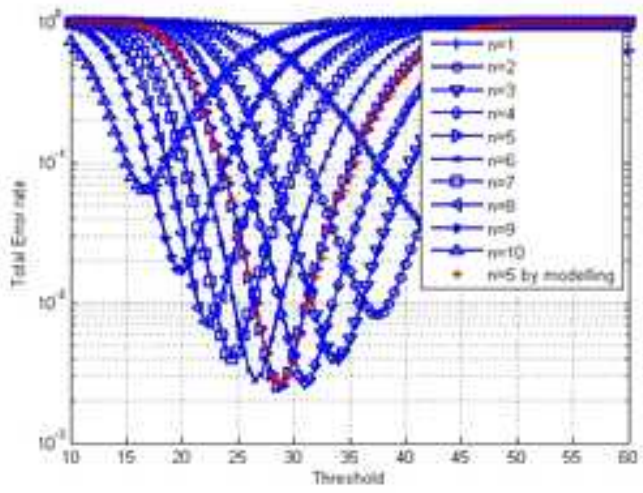

[Fig. 3] Total error probability $\left(Q_{\text {error }}\right)$ for $\mathrm{g}$ out of $\mathrm{k}=10 \mathrm{CRs}$ versus local threshold when ED is used locally with $\mathrm{SNR}=10 \mathrm{db}$ and $\mathrm{L}=10$ sensed samples used at each $\mathrm{CR}$.

Error on different no of CRs: In this section we are trying to find $Q_{\text {error }}$ at different number of SNR. Table 1 and Figure 4 shows the min $Q_{\text {error }}$ at different SNR. As per the analysis we have found that as we are increasing the SNR we get improvement in the total error rate: 


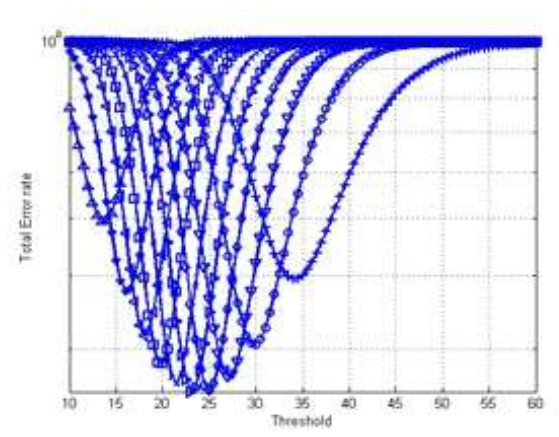

$\mathrm{SNR}=5 \mathrm{db}$

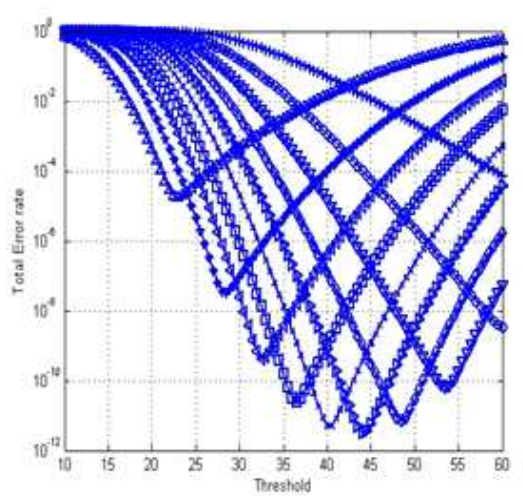

$S N R=15 d b$

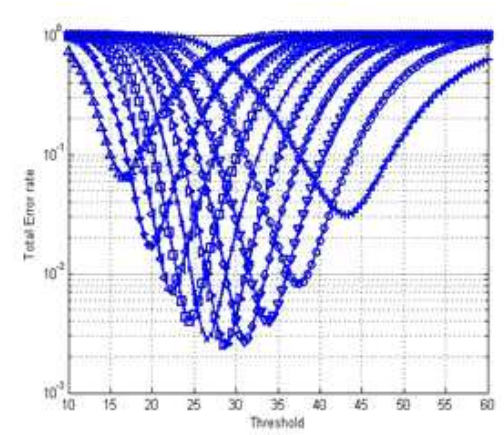

$\mathrm{SNR}=10 \mathrm{db}$

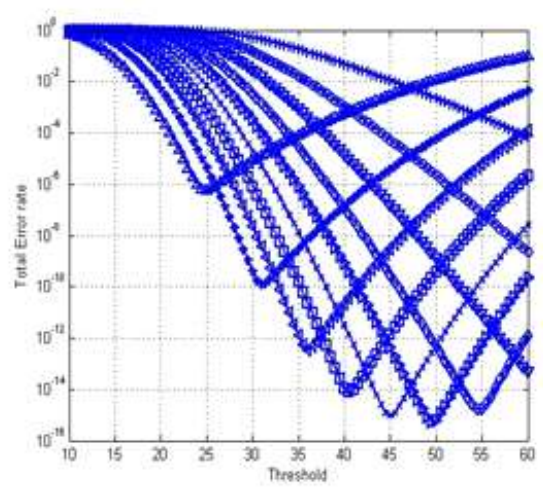

$\mathrm{SNR}=16 \mathrm{db}$

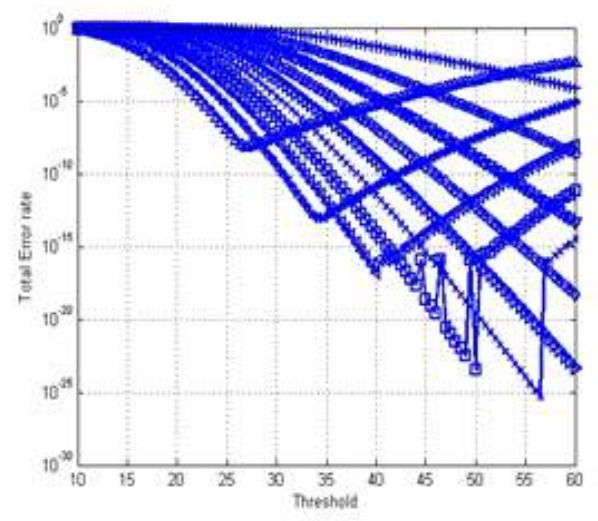

$\mathrm{SNR}=17 \mathrm{db}$

[Fig. 4] Total error probability $\left(Q_{\text {error }}\right)$ for $\mathrm{n}$ out of $\mathrm{K}=10 \mathrm{CRs}$ versus local threshold when $\mathrm{ED}$ is used locally with $\mathrm{SNR}=5,10,15,16,17, \mathrm{db}$ and $\mathrm{L}=10$ sensed samples used at each $\mathrm{CR}$. 
[Table 1] Min error rate at different value of snr

\begin{tabular}{|l|l|}
\hline SNR in $\mathrm{db}$ & Min error rate \\
\hline 5 & 1 \\
\hline 10 & 0.9995 \\
\hline 15 & 0.556 \\
\hline 16 & .0968 \\
\hline 17 & 0.0041 \\
\hline
\end{tabular}

From the above Figure 4, we can look that if we are increasing the SNR, the performance is increasing. Table 2 shows the optimal fusion rule and $\min$ when SNR is varied and the ED is used locally, with same number of the sensed samples (i.e. $\mathrm{L}=10$ ). The improvement in the performance by increasing the total number of user $\mathrm{K}$ for different SNR at CRs at fixed L, is noticeable. For example, $\min =0.2511$ when $\mathrm{SNR}=5 \mathrm{db}$ and $\mathrm{CRs}=4$ or 5 , and $\min =0.00251$ when SNR is increased to $10 \mathrm{db}$ and CRS $=5$. The increase in SNR causes decrease in the min $Q_{\text {error }}$ with variation in number of CRs.

[Table 2] Optimal number of $\mathrm{n}$ CRs for different SNR and its error level

\begin{tabular}{|l|l|l|}
\hline SNR in $\mathrm{db}$ & Error level & $\begin{array}{l}\text { Number of cognitive } \\
\text { user }\end{array}$ \\
\hline 5 & $10^{-0.6}$ & 4 or 5 \\
\hline 10 & $10^{-2.6}$ & 5 \\
\hline 13 & $10^{-6.4}$ & 5 or 6 \\
\hline 17 & $10^{-2.6}$ & 6 \\
\hline 18 & $10^{-3.4}$ & 8 \\
\hline 20 & $10^{-4.5}$ & 9 \\
\hline
\end{tabular}

\section{Conclusion}

We have calculated the performance of cooperative spectrum sensing with energy detection in cognitive radio networks. It is found that the optimal decision voting rule to minimize the total error probability is the half-voting rule. A method of numerically obtaining the optimal detection threshold has been presented. In addition, we have introduces an efficient optimization factor; the number of cognitive radio user, $\mathrm{K}$. As at each $\mathrm{CR}$ module we may have different SNR, depending upon the value of SNR we have derived the total error rate for different $\mathrm{n}(1$ to 10$)$. For SNR value $5,10,17,18,20$ we get $n$ optimal as 4 or 5,5 or 6 . 


\section{References}

[1] O. A. Alghamdi, M. A. Abu-Rgheff, and M. Z. Ahmed, MTM Parameters Optimization for 64-FFT Cognitive Radio Spectrum Sensing using Monte Carlo Simulation, in EMERGING 2010 : The Second International Conference on Emerging Network Intelligence, Florence-Italy, (2010), pp.107-113.

[2] E. C. Y. Peh, Y. C. Liang, Y. L. Guan and Y. Zeng Optimization of Cooperative Sensing in Cognitive Radio Networks: A Sensing-Throughput Tradeoff View, IEEE TRANSACTIONS ON VEHICULAR TECHNOLOGY, (2009), Vol.58, No.9.

[3] J. Shen, S. Liu, L. Zeng, G. Xie, J. Gao and Y. Liu, Optimisation of cooperative spectrum sensing in cognitive radio network, Communications, IET, (2009), Vol.3, pp.1170-1178.

[4] T. Yucek and H. Arslan, A survey of spectrum sensing algorithms for cognitive radio applications, Communications Surveys \& Tutorials, IEEE, (2009), Vol.11, pp.116-130.

[5] Z. Wei, R. K. Mallik and K. B. Letaief, Cooperative Spectrum Sensing Optimization in Cognitive Radio Networks, in Communications, 2008. ICC '08. IEEE International Conference on, (2008), pp.3411-3415.

[6] M. A. Abdulsattar, Energy detection technique for spectrum sensing in cognitive radio: a survey, Department of Electrical Engineering, University of Baghdad, Baghdad, Iraq, International Journal of Computer Networks \&Communications (IJCNC), (2012), Vol.4, No.5.

[7] B. Wang and K. Liu, Advances in cognitive radio networks: A survey, Selected Topics in Signal Processing, IEEE Journal, (2011), Vol.5, No.1, pp.5-23.

[8] E. Peh and L. Ying-Chang, Optimization for Cooperative Sensing in Cognitive Radio Networks, in Wireless Communications and Networking Conference, 2007. WCNC 2007. IEEE, (2007), pp. 27-32.

[9] Dong Won Kim, "Performance Analysis of Nonlinear Filters in Indoor Human Localization Based on Wireless Sensor Networks," JOURNAL OF PLATFORM TECHNOLOGY, VOL.3, NO. 2, JUNE 2015, pp.15-21

[10] Si-Yong Park, Sun-Myung Hwang, "A Congestion Avoidance Scheme based on modules of Energy harvesting Sensor Networks", Journal of Security Engineering, Vol.13, No.2 (2016), pp. 169-182, http://dx.doi.org/10.14257/jse.2016.04.08

[11] Si-Yong Park, Sun-Myung Hwang, "A Congestion Avoidance Policy to extend lifetime of Sensor Networks", Journal of Security Engineering, Vol.12, No.2 (2015), pp.169-180, http://dx.doi.org/10.14257/jse.2015.04.06

[12] Jae-Gon Yoo, Jong-Bae Kim, A Study Of Dangerous Driving Detection Application Using by Mobile Sensor,Asia-pacific Journal of Multimedia Services Convergent with Art, Humanities, and Sociology, ISSN:2383-5281, Vol.5, No.4, August (2015), pp.675-682, http://dx.doi.org/10.14257/AJMAHS.2015.08.33

[13] S. Madhavi, Secured Data Aggregation Scheduling in Ubiquitous Quantum Sensor Networks, Asia-pacific Journal of Multimedia Services Convergent with Art, Humanities, and Sociology, ISSN:2383-5281, Vol.4 No.1, June (2014), pp.17-30, http://dx.doi.org/10.14257/AJMAHS.2014.06.02

[14] S. Madhavi, Dynamic Distributed Power Adaption for Data Aggregation in Ubiquitous Sensor Networks, Asia-pacific Journal of Multimedia Services Convergent with Art, Humanities, and Sociology, 
ISSN:2383-5281, Vol.3 No.2, December (2013), pp.47-60, http://dx.doi.org/10.14257/AJMAHS.2013.12.04

[15] Sangho Lee, Haengrae Cho, Aekyung Moon, "Data Suppression Scheme for Corona based Wireless Sensor Networks", Journal of Security Engineering, Vol.11, No.4 (2014), pp.339-354, http://dx.doi.org/10.14257/jse.2014.08.06

[9] K. B. Letaief and W. Zhang, Cooperative communications for cognitive radio, Proc. IEEE, (2009), Vol.97, No.5, pp.878-893.

[10] S. Atapattu, C. Tellambura and H. Jiang, Energy detection based cooperative spectrum sensing in cognitive radio networks, IEEE Trans. Wireless Commun., (2011), Vol.10, No.4, pp.1232-1241.

[11] J. Ma and Y. Li, Soft combination and detection for cooperative spectrum sensing in cognitive radio networks, Proc. IEEE Global Telecomm. Conf., 2007, (2007) pp.3139- 3143.

[12] W. Zhang, R. K. Mallik and K. B. Letaief, Optimization of Cooperative Spectrum Sensing with Energy Detection in Cognitive Radio Networks, IEEE TRANSACTIONS ON WIRELESS COMMUNICATIONS, (2009), Vol.8, No.12.

[13] S. M. Kay, Fundamentals of Statistical Signal Processing and Estimation theory. Prentice Hall, (1998).

[14] A. Bagwari and G. S. Tomar, Cooperative Spectrum Sensing with Adaptive Double-Threshold Based Energy Detector in Cognitive Radio Networks, Springers International Journal of wireless personal Communication, (2013), Vol.73, No.3, pp.1005-19.

[15] A. Bagwari and G. S. Tomar, Cooperative Spectrum Sensing in MEDs Based CRNs Using Adaptive Double-Threshold scheme, Taylors and Francis - International Journal of Electronics, (2014), Vol.101, No.4, pp.37-41.

[16] A. Bagwari and G. S Tomar, Cooperative Spectrum Sensing with Multiple Antennas using Adaptive Double-Threshold Based Energy Detector in Cognitive Radio Networks, Springers Journal of Inst of Engg, India, Series B, (2014), Vol.95 No.2, pp.107-112.

[17] A. Bagwari and G. S. Tomar, Dual Detectors with Double Threshold for Spectrum Sensing in Cognitive Radio Networks, The Electronics World- The Essentials of Electronics Engineering Magazine, (2014), Vol.120, No.1937, pp.18-23.

[18] A. Bagwari and G. S. Tomar, Improved Spectrum Sensing technique using Multiple Energy Detectors for Cognitive radio networks, International Journal of Computer Applications, (2013), 62(4):11-21. 